\title{
The index for Fredholm elements in a Banach algebra via a trace
}

by

J. J. Grobler (Potchefstroom) and H. RAubenheimer (Johannesburg)

\begin{abstract}
We show that the existence of a trace on an ideal in a Banach algebra provides an elegant way to develop the abstract index theory of Fredholm elements in the algebra. We prove some results on the problem of the equality of the nonzero exponential spectra of elements $a b$ and $b a$ and use the index theory to formulate a condition guaranteeing this equality in a quotient algebra.
\end{abstract}

1. Introduction. The existence of a continuous trace on an operator ideal of operators on a Banach space has long been known to provide a useful tool for developing the Fredholm theory of operators (see for instance the monograph of A. Pietsch [20] and the paper by the present authors [13]). The problem of defining traces on ideals in a Banach algebra attracted the attention of many authors (see the papers by Puhl [22] and Aupetit and Mouton [2]). The main thrust of these papers was to show that a trace and a Fredholm determinant exist on the socle of a semisimple Banach algebra ([2] and [22]) and on the question of extending the trace or the determinant to larger ideals ([22] and [3]). The aim of this paper, on the other hand, is to present an axiomatic approach by assuming a trace to exist and then to show how useful such a trace is by applying it firstly to develop the index theory for abstract Fredholm elements in a semisimple Banach algebra (see the research notes of Barnes, Murphy, Smyth and West [5] and of Caradus, Pfaffenberger and Yood [10]). Here the commutative property of the trace provides some elegant proofs for the properties of the index. In the definitions and proofs we avoid using results from operator theory as far as possible.

An index function in a semisimple Banach algebra was also defined by Kraljević, Suljagić and Veselić (see [14]). We do not follow their approach, which is to define the trace of a Fredholm element $a$ as an element of $\mathbb{Z}^{n(a)}$

2000 Mathematics Subject Classification: Primary 46H05; Secondary 46H10, 47A10, 47A53.

Key words and phrases: trace, Fredholm element, index, exponential spectrum.

Support for the first-named author from the NRF is gratefully acknowledged. 
with $n(a) \in \mathbb{N}$, but we will show the connection of their trace with ours. Our definition of the index function relies on the existence of the trace function defined on an ideal containing the socle.

In the final section of our paper we give an application of the index function in terms of the exponential spectrum of a Banach algebra element. In this regard we refer to [19] where the exponential spectrum was studied in terms of the index group and an abstract index function.

2. Preliminaries and notation. All algebras in this paper are complex and unital. Denote by $A^{-1}$ the group of invertible elements in a Banach algebra $A$ and by

$$
\sigma(a, A)=\left\{\lambda \in \mathbb{C} \mid \lambda-a \notin A^{-1}\right\}
$$

the spectrum of $a \in A$. When no confusion can arise we write simply $\sigma(a)$ and the spectral radius of $a$ is denoted by $r(a)$. The connected component of $1 \in A^{-1}$ will be denoted by $\operatorname{Exp} A$. We recall that $\operatorname{Exp} A$ is a normal open and closed subgroup of $A^{-1}$ generated by the elements $e^{a}, a \in A$. The exponential spectrum of $a \in A$ is the set

$$
\varepsilon(a):=\varepsilon(a, A):=\{\lambda \in \mathbb{C} \mid \lambda-a \notin \operatorname{Exp} A\} .
$$

Let $[a, b]$ be the commutator $a b-b a$ of $a, b \in A$. By an ideal in $A$ we mean a two-sided ideal. An ideal $I$ in $A$ is said to be inessential [1, p. 106] if for every element $a$ in $I$ its spectrum in $A$ is either a finite set or a sequence converging to zero.

The radical of $A$ will be denoted by $\operatorname{Rad} A$, and $A$ is said to be semisimple if $\operatorname{Rad} A=\{0\}$. A Banach algebra $A$ is called semiprime if $0 \neq u \in A$ implies there exists $x \in A$ such that $u x u \neq 0$. All semisimple Banach algebras are semiprime. An element $a \in A$ is quasinilpotent if $\sigma(a)=\{0\}$. The set of these elements will be denoted by $\mathrm{QN}(A)$. Recall that if $I$ is a closed ideal in $A$ then $b \in A$ is called Riesz relative to $I$ if $b+I \in \mathrm{QN}(A / I)$ (see [5, Section R.1]). We denote by $\mathcal{R}(A, I)$ the set of Riesz elements relative to the ideal $I$. The set $\operatorname{kh}(I)$ is defined by $\operatorname{kh}(I):=\{b \in A \mid b+I \in \operatorname{Rad} A / I\}$. Clearly, this set is contained in $\mathcal{R}(A, I)$ and we have

$$
I \subset \operatorname{kh}(I) \subset \mathcal{R}(A, I) .
$$

An element $a \neq 0$ in a semiprime Banach algebra $A$ is called of rank one if there exists a linear functional $f_{a}$ on $A$ such that $a x a=f_{a}(x) a$ for all $x \in A$. For properties of these elements we refer to [22]. A minimal idempotent in $A$ is a nonzero idempotent $p$ such that $p A p$ is a division algebra. Minimal idempotents are examples of rank one elements [6, Proposition 31.3], and conversely, if $a$ is a rank one element, then $p=f_{a}(1)^{-1} a$ is easily seen to be a minimal idempotent. The finite rank elements of $A$, denoted by $\mathcal{F}(A)$, is the set of all $a \in A$ of the form $a=\sum_{i=1}^{n} a_{i}$ with each $a_{i}$ a rank one element. 
In the case of a semiprime Banach algebra, $\mathcal{F}$ coincides with the socle of $A$ (denoted by $\operatorname{Soc} A$ ), which is defined to be the sum of the minimal ideals in $A$. By [22, Lemma 2.7], $\mathcal{F}(A)$ is an ideal in $A$.

Another characterization of Soc $A$ can be found in [14] where the notion of degenerate elements in a semisimple Banach algebra is defined. An element $a$ is called degenerate if, for every $x \in A, d(a x)<\infty$ where $d(z)$ is defined to be the dimension of $\operatorname{Span}\left\{z, z^{2}, \ldots\right\}$. The set of all degenerate elements in $A$ is equal to $\operatorname{Soc} A$. In the same paper it is shown that if $\Gamma$ is the set of all similarity classes of minimal idempotents in Soc $A$ then the two-sided ideal $A_{\gamma}$ generated by an element $p_{\gamma} \in \gamma \in \Gamma$ is the same for any choice of $p_{\gamma} \in \gamma$. It is then shown that $\operatorname{Soc} A=\sum_{\gamma \in \Gamma} A_{\gamma}$.

The rank of an element in $\operatorname{Soc} A$ is defined in [14] as the supremum of $d(a x)$ as $x$ varies over $A$. This rank coincides with the spectral rank defined by Aupetit and Mouton in [2] to be

$$
\operatorname{rank}(a)=\sup _{x \in A} \#\{\sigma(x a) \backslash\{0\}\} \leq \infty,
$$

where $\# B$ denotes the number of elements in $B$. Aupetit and Mouton also showed [2, Corollary 2.18] that it is the smallest integer $k$ such that $a \in$ $I_{1}+\cdots+I_{k}$, with the $I_{j}$ distinct minimal left ideals. Of course, the rank of a rank one element is indeed one.

2.1. The trace. Let $I$ be an ideal in a Banach algebra $A$. A function $\tau: I \rightarrow \mathbb{C}$ is called a trace on $I$ if:

(TN) $\tau(p)=1$ for every rank one idempotent $p \in I$.

(TA) $\tau(a+b)=\tau(a)+\tau(b)$ for all $a, b \in I$.

(TH) $\tau(\alpha a)=\alpha \tau(a)$ for all $\alpha \in \mathbb{C}$ and $a \in I$.

(TC) $\tau(b a)=\tau(a b)$ for all $a \in I$ and $b \in A$.

We shall refer to an ideal on which a trace is defined as a trace ideal.

A trace $\tau$ on $I$ is called nilpotent if $\tau(a)=0$ for all nilpotent elements in $I$. We shall mainly concern ourselves with inessential ideals on which a trace is defined.

EXAMPLE 2.1. Let $X$ be a Banach space and let $T$ be a continuous linear operator on $X$ such that its range $Y:=R(T)$ is finite-dimensional. Let $\left\{x_{1}, \ldots, x_{n}\right\}$ be a basis for $Y$. Then there exist continuous linear functionals $\left\{x_{1}^{\prime}, \ldots, x_{n}^{\prime}\right\}$ on $X$ such that

$$
T x=\sum_{i=1}^{n} x_{i}^{\prime}(x) x_{i}=\left(\sum_{i=1}^{n} x_{i}^{\prime} \otimes x_{i}\right)(x) .
$$

The trace $\tau(T)$ of $T$ is defined as

$$
\tau(T)=\sum_{i=1}^{n} x_{i}^{\prime}\left(x_{i}\right)
$$


Note that this is the trace of the matrix $\left(x_{i}^{\prime}\left(x_{j}\right)\right)_{i, j=1}^{n}$, which, as can easily be checked, is the matrix representing the operator $T$ restricted to $Y$. Since this trace is independent of the choice of the basis, it is a well defined number. From the well known properties of the matrix trace it follows that conditions (TN), (TA), (TH) and (TC) above are satisfied for this trace on the ideal consisting of all operators with finite-dimensional range.

An example of a trace on the ideal of finite rank elements in a Banach algebra $A$ was provided by J. Puhl (see [22]). Let $A$ be a semiprime Banach algebra and let $0 \neq u \in A$ be a rank one element. J. Puhl defined a trace $\operatorname{tr}(u)$ of $u$ by

$$
u^{2}=\operatorname{tr}(u) u
$$

(see [22, Section 2]). It follows from the definition of rank one elements that $\operatorname{tr}(u)=f_{u}(1)$, and that $\operatorname{tr}(p)=1$ for every rank one idempotent $p$. If $u \in \operatorname{Soc} A$ and $u=\sum_{i=1}^{n} u_{i}$ with all the $u_{i}$ rank one elements then $\operatorname{tr}(u):=\sum_{i=1}^{n} \operatorname{tr}\left(u_{i}\right)$ [22, Definition 4.4] is well defined [22, Section 4]. It easily follows from the properties of a general trace $\tau$ on an inessential ideal $I$ that $\tau$ coincides with Puhl's trace on $I \cap \operatorname{Soc} A$. In fact, if $a$ is a rank one element, then, as we remarked earlier, $p=f_{a}(1)^{-1} a$ is a minimal idempotent. Consequently, $1=\tau(p)=f_{a}(1)^{-1} \tau(a)$. Hence, $\tau(a)=f_{a}(1)=\operatorname{tr}(a)$. By the linearity of $\tau$ it follows that $\tau(a)=\operatorname{tr}(a)$ for all $a \in \operatorname{Soc} A$. We also recall that Puhl showed that $\operatorname{tr}$ is nilpotent on $\operatorname{Soc} A$, and hence by what we have shown, any trace restricted to $\operatorname{Soc} A$ is also nilpotent.

Finally, we remark that B. Aupetit and H. du T. Mouton in [2] also defined a trace on Soc $A$ for $A$ a semisimple Banach algebra, by putting

$$
\operatorname{Tr}(a)=\sum_{\lambda \in \sigma(a)} m(\lambda, a) \lambda,
$$

where the number $m(\lambda, a)$ turns out to be the algebraic multiplicity of $\lambda$ for $a$. This trace is of course nilpotent on $\operatorname{Soc} A$ and it has the properties of an abstract trace. It is therefore equal to the Puhl trace and consequently coincides with any abstract trace defined on Soc $A$.

As shown by the definition of Aupetit and Mouton, the trace on finite rank elements is spectral, i.e., if $\lambda_{1}, \ldots, \lambda_{n}$ are the eigenvalues of $a$ each repeated according to its algebraic multiplicity, then

$$
\tau(a)=\sum_{i=1}^{n} \lambda_{i} .
$$

Since for every idempotent $p \in \operatorname{Soc} A$ we have $\sigma(p) \subseteq\{0,1\}$ we immediately see that $\tau(p) \in \mathbb{N}$ for every such $p$.

The fact that any abstract trace $\tau$ defined on $I$ corresponds to the Puhl trace on $\operatorname{Soc} A \cap I$ shows that $\tau$ can be extended to all elements of $\operatorname{Soc} A$. 
It can thus be extended to the ideal $I+\operatorname{Soc} A$. For this reason we will assume that if a trace is defined on an ideal $I$, then $I$ includes Soc $A$.

3. Fredholm elements and index theory. We start off in a very general setting in which the assumptions on the trace are minimal (in particular, we do not assume the trace ideal to be closed).

Let $I$ be a proper ideal of the Banach algebra $A$ and suppose that $\tau$ : $I \rightarrow \mathbb{C}$ is a trace defined on $I$. The following definition is a generalization of Definitions F.2.5 and F.3.12 in [5] as stated on page 49 of [5].

Definition 3.1. We call the element $a \in A$ a Fredholm element relative to $I$ if there exists an element $a_{0} \in A$ such that

(i) $a a_{0}-1 \in I$;

(ii) $a_{0} a-1 \in I$.

The set of all Fredholm elements relative to $I$ is denoted by $\Phi(A, I)$.

Clearly, $a \in \Phi(A, I)$ if and only if $\bar{a}=a+I$ is invertible in the quotient algebra $A / I$. Also, $A^{-1} \subset \Phi(A, I)$ and $\Phi(A, I)$ is a multiplicative semigroup. Note that since we do not assume $I$ to be closed, $A / I$ will not be a Banach algebra in general.

ExAmple 3.2. Let $X$ be a Banach space. The operator $T$ on $X$ is called a Fredholm operator if the null spaces $N(T)$ and $N\left(T^{\prime}\right)$ of both $T$ and its adjoint $T^{\prime}$ are finite-dimensional and if the range $R(T)$ of $T$ is closed (see [23, Chapter V]). Its index $\iota(T)$ is defined to be

$$
\iota(T)=\operatorname{dim}(N(T))-\operatorname{dim}\left(N\left(T^{\prime}\right)\right)=\alpha-\beta .
$$

For a Fredholm operator $T$ on $X$ there exists an operator $T_{0}$ on $X$ such that

$$
T_{0} T=I-F_{1} \quad \text { and } \quad T T_{0}=I-F_{2},
$$

where $F_{1}$ is the projection of $X$ onto $N(T)$ and $F_{2}$ the projection of $X$ onto a finite-dimensional subspace $X_{0} \subset X$ satisfying $X=R(T) \oplus X_{0}$ and $\operatorname{dim}\left(X_{0}\right)=\beta$ ([23, Theorem V.1.4]). Since $F_{1}$ has finite-dimensional range, it is of the form $\sum_{i=1}^{\alpha} x_{i}^{\prime} \otimes x_{i}$, and since it is a projection, $x_{i}^{\prime}\left(x_{j}\right)=\delta_{i j}$. It follows that its trace $\tau\left(F_{1}\right)$ satisfies $\tau\left(F_{1}\right)=\alpha$. Similarly, $\tau\left(F_{2}\right)=\operatorname{dim}\left(X_{0}\right)=\beta$. Hence,

$$
\iota(T)=\alpha-\beta=\tau\left(F_{1}-F_{2}\right)=\tau\left(T T_{0}-T_{0} T\right) .
$$

Following the example, we define an index on $\Phi(A, I)$ with the aid of the trace as follows.

Definition 3.3. Let $\tau$ be a trace on the ideal $I$ in $A$. We define the index function $\iota: \Phi(A, I) \rightarrow \mathbb{C}$ by

$$
\iota(a):=\tau\left(a a_{0}-a_{0} a\right)=\tau\left(\left[a, a_{0}\right]\right) \quad \text { for all } a \in \Phi(A, I),
$$

where $a_{0} \in A$ satisfies $a a_{0}-1 \in I$ and $a_{0} a-1 \in I$. 
Proposition 3.4. The index function is well defined on $\Phi(A, I)$.

Proof. We note firstly that $a a_{0}-a_{0} a=a a_{0}-1-\left(a_{0} a-1\right) \in I$, and so $\tau\left(a a_{0}-a_{0} a\right)$ exists. Next, let $a_{0}^{\prime} \in A$ also satisfy $a a_{0}^{\prime}-1 \in I$ and $a_{0}^{\prime} a-1 \in I$. Then we have $\tau\left(a a_{0}^{\prime}-a_{0}^{\prime} a\right)-\tau\left(a a_{0}-a_{0} a\right)=\tau\left(a\left(a_{0}^{\prime}-a_{0}\right)-\left(a_{0}^{\prime}-a_{0}\right) a\right)$, and since $a_{0}^{\prime} \equiv a_{0} \bmod I$, both terms in the argument are in $I$. By the properties of a trace we therefore get

$$
\tau\left(a a_{0}^{\prime}-a_{0}^{\prime} a\right)-\tau\left(a a_{0}-a_{0} a\right)=\tau\left(a\left(a_{0}^{\prime}-a_{0}\right)\right)-\tau\left(\left(a_{0}^{\prime}-a_{0}\right) a\right)=0
$$

and so $\iota$ is well defined.

The definition of Fredholm elements is symmetric. Therefore, if $a \in$ $\Phi(A, I)$ then every element $a_{0}$ satisfying the definition is also in $\Phi(A, I)$ and its index satisfies $\iota\left(a_{0}\right)=-\iota(a)$. Also, if $a$ is invertible we can take $a_{0}=a^{-1}$ and we get $\iota(a)=0$.

Proposition 3.5. For $a, b \in \Phi(A, I)$ we have $\iota(a b)=\iota(a)+\iota(b)$.

Proof. As observed above, $a b \in \Phi(A, I)$ for $a, b \in \Phi(A, I)$. Let $a_{0}, b_{0} \in A$ satisfy $a a_{0}-1, a_{0} a-1 \in I$ and $b b_{0}-1, b_{0} b-1 \in I$. It is easily seen that

$$
(a b)\left(b_{0} a_{0}\right)-1 \in I, \quad\left(b_{0} a_{0}\right)(a b)-1 \in I, \quad b\left(b_{0} a_{0}\right) a-1 \in I .
$$

Therefore,

$$
\begin{aligned}
\iota(a b) & =\tau\left((a b)\left(b_{0} a_{0}\right)-\left(b_{0} a_{0}\right)(a b)\right) \\
& =\tau\left((a b)\left(b_{0} a_{0}\right)-b\left(b_{0} a_{0}\right) a\right)+\tau\left(b\left(b_{0} a_{0}\right) a-\left(b_{0} a_{0}\right)(a b)\right) \\
& =\tau\left(a\left(b b_{0} a_{0}\right)-\left(b b_{0} a_{0}\right) a\right)+\tau\left(b\left(b_{0} a_{0} a\right)-\left(b_{0} a_{0} a\right) b\right)=\iota(a)+\iota(b) .
\end{aligned}
$$

COROllary 3.6. The index function $\iota$ is a semigroup homomorphism of $\Phi(A, I)$ into the additive group of $\mathbb{C}$.

We gather some further properties of the index function.

Proposition 3.7. Let $a \in \Phi(A, I)$.

(i) For every $q \in I$ we have $\iota(a+q)=\iota(a)$.

(ii) For every $\lambda \neq 0$ we have $\lambda a \in \Phi(A, I)$ and $\iota(\lambda a)=\iota(a)$.

(iii) For every $\lambda \neq 0$ and every $q \in I$ we have $\iota(\lambda-q)=0$.

(iv) The set $\Phi(A, I)$ is open in $A$.

(v) The index function $\iota$ is constant on every component of $\Phi(A, I)$.

(vi) The index function $\iota: \Phi(A, I) \rightarrow \mathbb{C}$ is continuous.

Proof. (i) With $a_{0}$ as before and for $q \in I$ we have $(a+q) a_{0}-1=$ $\left(a a_{0}-1\right)+q a_{0} \in I$ and likewise $a_{0}(a+q)-1 \in I$. Hence, $a+q \in \Phi(A, I)$ and $\iota(a+q)=\tau\left((a+q) a_{0}-a_{0}(a+q)\right)=\tau\left(a a_{0}-a_{0} a\right)+\tau\left(q a_{0}-a_{0} q\right)=\iota(a)$.

(ii) For $\lambda \neq 0$ we have $(\lambda a)\left(\lambda^{-1} a_{0}\right)-1 \in I$ and $\left(\lambda^{-1} a_{0}\right)(\lambda a)-1 \in I$. It follows immediately that $\iota(\lambda a)=\iota(a)$.

(iii) This follows immediately from (i) and (ii) since $\iota(1)=0$. 
(iv) Let $a \in \Phi(A, I)$ and let $a a_{0}-1=p \in I$ and $a_{0} a-1=q \in I$. Let $b \in A$ satisfy $\|a-b\|<\left\|a_{0}\right\|^{-1}$. It follows from $b a_{0}=1+p-(a-b) a_{0}=$ $p+\left(1-(a-b) a_{0}\right)$, and the fact that $u:=1-(a-b) a_{0}$ is invertible, that $b a_{0} u^{-1}-1=p u^{-1} \in I$. Similarly, we get $v^{-1} a_{0} b-1=v^{-1} q \in I$ where $v:=1-a_{0}(a-b)$. By the series expansion for $u^{-1}$ and $v^{-1}$ we find that $a_{0} u^{-1}=v^{-1} a_{0}$ and this shows by definition that $b \in \Phi(A, I)$. Hence, $\Phi(A, I)$ is open.

(v) Using the argument and notation in (iv) we find for all $b$ in a neighbourhood of $a$ that $b b_{0}-1 \in I$ and $b_{0} b-1 \in I$ with $b_{0}=a_{0} u^{-1}$. It follows that

$$
\iota(b)=-\iota\left(b_{0}\right)=-\iota\left(a_{0}\right)-\iota\left(u^{-1}\right)=-\iota\left(a_{0}\right)=\iota(a) .
$$

(vi) This follows immediately from (iv) and (v).

Corollary 3.8. Let $(A / I)^{-1}$ denote the group of invertible elements of $A / I$. Then $\bar{\iota}:(A / I)^{-1} \rightarrow \mathbb{C}$ defined by $\bar{\iota}(a+I)=\iota(a)$ is a group homomorphism.

It seems as though this is the furthest one can develop the index theory in the general case. To proceed we need the following definition.

Definition 3.9. An idempotent $p$ is called a left Barnes idempotent for $a \in A$ if

$$
a A=(1-p) A,
$$

and a right Barnes idempotent for $a \in A$ if

$$
A a=A(1-q) .
$$

Also, for $a \in A$ we define the right and left annihilators respectively as the sets

$$
N_{r}(a):=\{x \in A \mid a x=0\}, \quad N_{\ell}(a):=\{x \in A \mid x a=0\} .
$$

As we shall see shortly, a Barnes idempotent belonging to a given element $a \in A$ is not uniquely determined. We note the following facts (see [5, F.1.9]).

Proposition 3.10. Let $A$ be a unital Banach algebra. If $p$ is a left Barnes idempotent for a then $A p=N_{\ell}(a)$, and in particular, $p \in N_{\ell}(a)$. Similarly, if $q$ is a right Barnes idempotent for $a \in A$ then $q A=N_{r}(a)$ and so $q \in N_{r}(a)$.

Proof. Let $p$ be a left Barnes idempotent for $a$. By (3.1) there exists some $b \in A$ such that $a=(1-p) b$. Hence, $p a=p(1-p) b=0$ and so $p \in N_{\ell}(a)$. It follows that $A p \subset N_{\ell}(a)$. On the other hand, again by (3.1) we have $1-p=a b$ for some $b \in A$. So, if $x \in N_{\ell}(a)$, we have $x(1-p)=x a b=0$. Thus, $x=x p \in A p$. This establishes $A p=N_{\ell}(a)$.

THEOREM 3.11. Let $A$ be a semisimple unital Banach algebra and let the trace ideal I satisfy $\operatorname{Soc} A \subset I \subset \operatorname{kh}(\operatorname{Soc} A)$. Then: 
(i) $a \in \Phi(A, I)$ if and only if there exist left and right Barnes idempotents $p$ and $q$, respectively, in $\operatorname{Soc} A$ and an element $a_{0} \in A$ such that

$$
a a_{0}=1-p \quad \text { and } \quad a_{0} a=1-q .
$$

(ii) $\iota(a) \in \mathbb{Z}$.

Proof. (i) Since $A$ is a semisimple unital Banach algebra it follows that Soc $A$ is nontrivial. As we remarked earlier, we may assume that the trace ideal $I$ contains $\operatorname{Soc} A$. Since an element $a$ is invertible modulo $\operatorname{Soc} A$ if and only it is invertible modulo $\mathrm{kh}(\operatorname{Soc} A)$ (see [5, Theorem BA.2.4, p. 103]), we have $\Phi(A, I)=\Phi(A, \operatorname{Soc} A)$. It then follows from [5, Theorem F.1.10] that $a \in \Phi(A, I)$ if and only if there exist left and right Barnes idempotents in the socle for $a$. Let $p \in \operatorname{Soc} A \subset I$ be a left Barnes idempotent for $a$; then there exists by (3.1) an element $a_{0} \in A$ such that $a a_{0}=1-p$ and also an element $b \in A$ such that $a=(1-p) b$. The latter relation implies that $(1-p) a=a$. Hence, $a_{0} a$ is an idempotent, because

$$
\left(a_{0} a\right)^{2}=a_{0}\left(a a_{0}\right) a=a_{0}(1-p) a=a_{0} a .
$$

Let $q:=1-a_{0} a$; since $a \in \Phi(A, I)$ and the equivalence class containing $a_{0}$ is a right inverse of $a$ modulo $I=\operatorname{Soc} A$, it is also a left inverse of $a$ modulo $I$. It follows that $q \in I=$ Soc $A$ and $a q=a\left(1-a_{0} a\right)=a-a a_{0} a=a-(1-p) a=0$ and so $a=a(1-q)$. The two equalities

$$
1-q=a_{0} a \quad \text { and } \quad a=a(1-q)
$$

together imply that $A a=A(1-q)$ and so $q$ is a right Barnes idempotent for $a$. Hence, (i) holds.

(ii) This follows immediately from (3.3) because

$$
\iota(a)=\tau\left(a a_{0}-a_{0} a\right)=\tau(1-p-(1-q))=\tau(q-p)=\tau(q)-\tau(p) .
$$

But, as remarked earlier, $\tau$ is a spectral trace on $\operatorname{Soc} A$ and consequently $\tau(p), \tau(q) \in \mathbb{N}$. Thus, $\iota(a) \in \mathbb{Z}$.

Assumption. For the remainder of this section we assume that $A$ is a unital semisimple Banach algebra.

Definition 3.12. For a Fredholm element $a$ we call a right and left Barnes idempotent $p$ and $q$ that satisfy (3.3) associated.

Note. We may assume without loss of generality that the element $a_{0}$ which satisfies (3.4) has the properties that $a_{0}(1-p)=a_{0}$ and $(1-q) a_{0}=a_{0}$ (else we replace $a_{0}$ by $(1-q) a_{0}(1-p)$ ).

Corollary 3.13. Let a be a Fredholm element of $A$. Then there exist associated Barnes idempotents $p$ and $q$ such that $\iota(a)=\tau(q)-\tau(p)$.

We now show that the traces of all left (resp. right) Barnes idempotents belonging to an element $a \in A$ are equal. 
THEOREM 3.14. Let $p$ and $q$ be respectively left and right Barnes idempotents of the Fredholm element $a \in A$. Then $\tau(p)$ is equal to the cardinality of a maximal set of orthogonal minimal idempotents in $N_{r}(a)=A p$. Similarly, $\tau(q)$ is equal to the cardinality of a maximal set of orthogonal minimal idempotents in $N_{\ell}(a)=q A$.

Proof. Since $N_{\ell}=N_{\ell}(a)=A p$ is a left ideal contained in the socle of $A$, we deduce by [5, Lemma F.1.7] that every orthogonal subset of minimal idempotents in $N_{\ell}$ is finite. Let $\left\{e_{1}, \ldots, e_{k}\right\}$ be a maximal subset of minimal idempotents; then $N_{\ell}=\sum_{i=1}^{k} A e_{i}$ and we can write

$$
p=x_{1} e_{1}+\cdots+x_{k} e_{k} .
$$

Again by [5, Lemma F.1.7], $\bar{p}=e_{1}+\cdots+e_{k}$ is an idempotent in $N_{\ell}(a)$ and $N_{\ell}(a)=A \bar{p}$. Note now that $p \bar{p}=p$. We then have

$$
a a_{0}=(1-\bar{p}) a a_{0}=(1-\bar{p})(1-p)=1-\bar{p}-p+\bar{p} p
$$

and it follows from the commutativity property of the trace that

$$
\begin{aligned}
\tau(p) & =\tau\left(1-a a_{0}\right)=\tau(\bar{p}+p-\bar{p} p)=\tau(\bar{p})+\tau(p)-\tau(p \bar{p}) \\
& =\tau(\bar{p})+\tau(p)-\tau(p)=\tau(\bar{p})=\tau\left(e_{1}\right)+\cdots+\tau\left(e_{k}\right)=k .
\end{aligned}
$$

The proof for a right Barnes idempotent $q$ is entirely similar.

REMARK. We note two interesting facts which follow from the proof. In the first place, since the $e_{i}$ are minimal idempotents, we have $e_{i} x_{i} e_{i}=\lambda_{i} e_{i}$ for some $\lambda_{i} \in \mathbb{C}$. So by the commutativity of the trace,

$$
\tau(p)=\sum_{i=1}^{k} \tau\left(x_{i} e_{i}\right)=\sum_{i=1}^{k} \tau\left(e_{i} x_{i} e_{i}\right)=\sum_{i=1}^{k} \tau\left(\lambda_{i} e_{i}\right)=\sum_{i=1}^{k} \lambda_{i} .
$$

Secondly, for every left Barnes idempotent $p$ for $a$, we see that $p+\bar{p}-\bar{p} p$ (with the notation of the proof) is again a left Barnes idempotent for $a$. Similarly, if $q$ is the right Barnes idempotent for $a$ associated with $p$, then $q+\bar{q}-q \bar{q}$ is again a right Barnes idempotent for $a$ and it is associated to $p+\bar{p}-\bar{p} p$.

Corollary 3.15. Let a be a Fredholm element of $A$ with left Barnes idempotent $p$. Then $\tau(p)=\operatorname{rank}(p)$. Similarly, for a right Barnes idempotent $q$ we have $\tau(q)=\operatorname{rank}(q)$.

Proof. This follows immediately from [2, Corollary 2.18] as we already remarked in our introduction.

The fact that for any left Barnes idempotent $p$ for $a$ the number $\tau(p)$ is uniquely defined, even though the Barnes idempotent is not, enables us to define the nullity and deficiency of an element $a \in \Phi(A, I)$ as follows. 
Definition 3.16. The nullity $n(a)$ and deficiency $d(a)$ of $a \in \Phi(A, I)$ are defined as

$$
n(a):=\tau(q) \text { and } \quad d(a):=\tau(p),
$$

where $q$ is a right Barnes idempotent and $p$ a left Barnes idempotent of $a$.

We have

Theorem 3.17. Let $A$ be a unital Banach algebra and let the trace ideal $I$ satisfy $\operatorname{Soc} A \subset I \subset \mathrm{kh}(\operatorname{Soc} A)$. Then for every $a \in \Phi(A, I)$ we have

$$
\iota(a)=n(a)-d(a)
$$

and

$$
A^{-1}=\{a \in \Phi(A, I) \mid n(a)=d(a)=0\} .
$$

Proof. If $a \in A$ is invertible, then 0 is a left as well as right Barnes idempotent for $a$ and so $n(a)=d(a)=0$. On the other hand, $\tau(p) \geq 1$ for every nonzero left Barnes idempotent of $a$. If, therefore, the condition $n(a)=d(a)=0$ holds, we see that $a$ has a right and left inverse and hence belongs to $A^{-1}$.

We recall that two elements $a, b \in A$ are called similar if there exists an element $u \in A^{-1}$ such that $a=u^{-1} b u$. Let $\Gamma$ be the set of similarity classes of minimal idempotents in Soc $A$. In [14] H. Kraljević, S. Suljagić and K. Veselić proved that Soc $A=\sum_{\gamma \in \Gamma} A_{\gamma}$, where $A_{\gamma}$ is the two-sided ideal generated by $p \in \gamma \in \Gamma$ and the sum is an algebraic direct sum ([14, Theorem 2.12]). So, for every $a \in \operatorname{Soc} A$ there exists a finite set $\Gamma_{a} \subset \Gamma$ such that $a=\sum_{\gamma \in \Gamma_{a}} a_{\gamma}$. If we put $a_{\gamma}=0$ if $\gamma \in \Gamma \backslash \Gamma_{a}$, we have $a=\sum_{\gamma \in \Gamma} a_{\gamma}$.

Let $p$ and $q$ be left and right Barnes idempotents respectively and suppose that

$$
p=\sum_{\gamma \in \Gamma} p_{\gamma}, \quad q=\sum_{\gamma \in \Gamma} q_{\gamma} .
$$

LEMMA 3.18. The nonzero elements $p_{\gamma}$ and $q_{\gamma}$ are minimal idempotents, and consequently rank one elements. Hence, $\tau\left(p_{\gamma}\right)=\operatorname{rank}\left(p_{\gamma}\right)$ and $\tau\left(q_{\gamma}\right)=$ $\operatorname{rank}\left(q_{\gamma}\right)$.

Proof. We prove this for $p_{\gamma}$. Note firstly that if $\gamma \neq \xi \in \Gamma$, then $p_{\gamma} p_{\xi}=0$, because the product belongs to $A_{\gamma} \cap A_{\xi}=\{0\}$. Hence, $p p_{\gamma}=p_{\gamma}^{2}$. Suppose now that $p_{\gamma}=x_{\gamma} e_{\gamma} y_{\gamma}$ with $x_{\gamma}, y_{\gamma} \in A$ and $e_{\gamma} \in \gamma$. Then, since $e_{\gamma}$ is a minimal idempotent,

$$
p_{\gamma}^{2}=\left(x_{\gamma} e_{\gamma} y_{\gamma}\right)\left(x_{\gamma} e_{\gamma} y_{\gamma}\right)=x_{\gamma}\left(e_{\gamma}\left(y_{\gamma} x_{\gamma}\right) e_{\gamma}\right) y_{\gamma}=x_{\gamma}\left(\lambda_{\gamma} e_{\gamma}\right) y_{\gamma}=\lambda_{\gamma} p_{\gamma} .
$$

It follows that

$$
\left(\lambda_{\gamma}-p\right) p_{\gamma}=\lambda_{\gamma} p_{\gamma}-\left(\sum_{\xi \in \Gamma} p_{\xi}\right) p_{\gamma}=\lambda_{\gamma} p_{\gamma}-p_{\gamma}^{2}=0
$$


and so $\lambda_{\gamma}-p$ is not invertible, i.e., $\lambda_{\gamma} \in \sigma(p)$. But since $p$ is a projection, $\sigma(p) \subseteq\{0,1\}$ and so, since $A$ is semisimple, $\lambda_{\gamma}=1$. Hence, $p_{\gamma}$ is an idempotent belonging to $A_{\gamma}$. It then follows from $A p_{\gamma} A \subset A_{\gamma}$ that $p_{\gamma}$ is a minimal idempotent.

In [14] H. Kraljević, S. Suljagić and K. Veselić then defined, for a Fredholm element $a \in \Phi(A$, Soc $A)$ with Barnes left and right Barnes idempotents $p$ and $q$ respectively,

$$
\operatorname{Ind}_{\gamma}(a)=\operatorname{rank}\left(q_{\gamma}\right)-\operatorname{rank}\left(p_{\gamma}\right)=\tau\left(q_{\gamma}\right)-\tau\left(p_{\gamma}\right)
$$

and the index of $a$ as

$$
\operatorname{Ind}(a)=\left(\operatorname{Ind}_{\gamma}(a)\right)_{\gamma \in \Gamma} \in \mathbb{Z}^{\Gamma} .
$$

It is clear from the lemma above that we have

$$
\iota(a)=\tau(q)-\tau(p)=\sum_{\gamma \in \Gamma}\left(\tau\left(q_{\gamma}\right)-\tau\left(p_{\gamma}\right)\right)=\sum_{\gamma \in \Gamma} \operatorname{Ind}_{\gamma}(a) .
$$

The advantage of the index $\operatorname{Ind}(a) \in \mathbb{Z}^{\Gamma}$ is that the next theorem can be formulated exactly as in the operator case (see [14, Theorem 7.6]). A disadvantage is that the definition is not as intrinsic as our definition: one first has to find Barnes idempotents in order to define $\operatorname{Ind}_{\gamma}$. We formulate the theorem in our notation and supply an easy short proof.

TheOREM 3.19. Let $A$ be a unital semisimple Banach algebra and let I be a closed trace ideal in $A$ such that $\operatorname{Soc} A \subset I \subset \mathrm{kh}(\operatorname{Soc} A)$. Let a $\in \Phi(A, I)$ and let $\Gamma$ be the set of similarity classes of minimal idempotents in $\operatorname{Soc} A$. Let $p$ and $q$ be respectively left and right Barnes idempotents for a and let $p=\sum_{\gamma} p_{\gamma}$ and $q=\sum_{\gamma} q_{\gamma}$. Then the following are equivalent:

(i) $\tau\left(p_{\gamma}\right)=\tau\left(q_{\gamma}\right)$ for every $\gamma \in \Gamma$, i.e., $\operatorname{Ind}(a)=0$.

(ii) There exists some $u \in A^{-1}$ and some $d \in \operatorname{Soc} A$ such that $a=u+d$.

Proof. (ii) $\Rightarrow$ (i). Let $a_{0}$ be an inverse of $a$ modulo Soc $A$ satisfying

$$
a a_{0}=1-p \quad \text { and } \quad a_{0} a=1-q .
$$

It follows from (ii) that $v:=u^{-1}$ is also an inverse of $a$ modulo Soc $A$ and so $a_{0}=v+e$ for some $e \in \operatorname{Soc} A$. Substitution in (3.5) then yields

$$
-p=d v+a e \text { and }-q=v d+e a .
$$

So, if $d=\sum_{\gamma} d_{\gamma}$ and $e=\sum_{\gamma} e_{\gamma}$, then for every $\gamma \in \Gamma$ we have

$$
-p_{\gamma}=d_{\gamma} v+a e_{\gamma} \quad \text { and } \quad-q_{\gamma}=v d_{\gamma}+e_{\gamma} a
$$

and (i) follows.

(i) $\Rightarrow$ (ii). The assumption implies that $p_{\gamma} \neq 0$ iff $q_{\gamma} \neq 0$ for all $\gamma \in \Gamma$. Let $u_{\gamma} \in A^{-1}$ be such that if $p_{\gamma} \neq 0$ then $u_{\gamma} p_{\gamma}=q_{\gamma} u_{\gamma}$. Let $u:=\sum_{\gamma} u_{\gamma} p_{\gamma}=$ $\sum_{\gamma} q_{\gamma} u_{\gamma}$. Let $v:=\sum_{\gamma} p_{\gamma} u_{\gamma}^{-1}=\sum_{\gamma} u_{\gamma}^{-1} q_{\gamma}$. Observe that every term is in $A_{\gamma}$ and so it follows that $v u=p$ and $u v=q$. Finally observe that if $a_{0}$ is 
such that (3.5) holds, then $u=u p=q u \in N_{\ell}(a) \cap N_{r}(a)$ and $v=p v=$ $v q \in N_{\ell}\left(a_{0}\right) \cap N_{r}\left(a_{0}\right)$. If we write $a=(a+v)-v$, then $d=-v \in \operatorname{Soc} A$ and $a+v \in A^{-1}$ because

$$
\begin{aligned}
& (a+v)\left(a_{0}+u\right)=a a_{0}+v a_{0}+a u+v u=1-p+p=1, \\
& \left(a_{0}+u\right)(a+v)=a_{0} a+u a+a_{0} v+u v=1-q+q=1 .
\end{aligned}
$$

Let $X$ and $Y$ be Banach spaces and let $B: X \rightarrow Y$ and $A: Y \rightarrow X$ be bounded linear operators. There is a classical result that if $I-A B$ is a Fredholm operator then $\iota(I-A B)=\iota(I-B A)$ (see [4, Theorem 6]). We prove the analogue in general.

TheOREM 3.20. Let $A$ be a unital semisimple Banach algebra and let $I$ be a closed trace ideal in $A$ such that $\operatorname{Soc} A \subset I \subset \operatorname{kh}(\operatorname{Soc} A)$. If $a, b \in A$ with $1-a b \in \Phi(A, I)$ then $\iota(1-a b)=\iota(1-b a)$.

Proof. Let $p$ and $q$ be respectively right and left Barnes idempotents of $1-a b \in \Phi(A, I)$ such that $\iota(1-a b)=\tau(q)-\tau(p)$ (see Corollary 3.13). From the definition of a right Barnes idempotent (Definition 3.9), there exists an element $x \in A$ such that

$$
(1-a b) x=1-p \quad \text { and } \quad x(1-a b)=1-q .
$$

If $x_{0}=b x a+1$ then, using (3.6), we get

$$
\begin{aligned}
(1-b a) x_{0} & =b x a+1-b(a b x) a-b a \\
& =b x a+1-b(x-1+p) a-b a=1-b p a, \\
x_{0}(1-b a) & =b x a-b(x a b) a+1-b a \\
& =b x a-b(x-1+q) a+1-b a=1-b q a .
\end{aligned}
$$

This shows that $1-b a \in \Phi(A, I)$ and applying Definition 3.3 to (3.7)-(3.8), we have

$$
\begin{aligned}
\iota(1-b a) & =\tau\left((1-b a) x_{0}-x_{0}(1-b a)\right)=\tau(1-b p a-(1-b q a)) \\
& =\tau(b q a)-\tau(b p a) \quad \text { by }(\mathrm{TA}) .
\end{aligned}
$$

But $\tau(b q a)=\tau(a b q)=\tau(a b q-q+q)=\tau((a b-1) q+q)=\tau(q)$, since $q \in N_{r}(1-a b)$, and similarly, because $p \in N_{\ell}(1-a b)$ we have $\tau(b p a)=\tau(p)$. It follows that $\iota(1-b a)=\tau(q)-\tau(p)=\iota(1-a b)$.

The punctured neighbourhood theorem also follows.

TheOREM 3.21. Let $A$ be a unital Banach algebra and let the trace ideal $I$ satisfy $\operatorname{Soc} A \subset I \subset \operatorname{kh}(\operatorname{Soc} A)$. Then for every $a \in \Phi(A, I)$ there exists $\epsilon>0$ such that, for all $\lambda$ satisfying $0<|\lambda|<\epsilon, \lambda-a$ is in $\Phi(A, I)$ and

(i) $n(\lambda-a)$ is constant and $n(\lambda-a) \leq n(a)$;

(ii) $d(\lambda-a)$ is constant and $d(\lambda-a) \leq d(a)$;

(iii) $\iota(\lambda-a)$ is constant and $\iota(\lambda-a)=\iota(a)$. 
Proof. Since $\lambda-a$ is in an $\epsilon$-neighbourhood of $-a$ for all $0<|\lambda|<\epsilon$ it follows, from the fact that $\Phi(A, I)$ is open, that for $\epsilon$ properly chosen $\lambda-a \in \Phi(A, I)$ and also that $\iota(\lambda-a)=\iota(a)$.

Suppose now that $a_{0} a=1-q$, with $q$ a right Barnes idempotent for $a$ where we suppose, as we may, that $q a_{0}=0$. If $|\lambda|<1 /\left\|a_{0}\right\|$ then $\lambda a_{0}-1$ is invertible and so we deduce from $a_{0}(\lambda-a)=q+\left(\lambda a_{0}-1\right)$ that, for all $|\lambda|<1 /\left\|a_{0}\right\|$,

$$
\left[\left(\lambda a_{0}-1\right)^{-1} a_{0}\right](\lambda-a)=1-\left(1-\lambda a_{0}\right)^{-1} q .
$$

Since $q a_{0}=0$, we have $q\left(1-\lambda a_{0}\right)^{-1}=q \sum_{k=0}^{\infty}\left(\lambda a_{0}\right)^{k}=q$ and this implies that the element $q(\lambda):=\left(1-\lambda a_{0}\right)^{-1} q$ is an idempotent. In fact, it is a Barnes idempotent for $a_{0}(\lambda-a)$ because it follows from equation (3.9) that $q(\lambda) \in N_{r}\left(a_{0}(\lambda-a)\right)$. Hence, $N_{r}(\lambda-a) \subset N_{r}\left(a_{0}(\lambda-a)\right)=q(\lambda) A$ implies that

$$
n(\lambda-a) \leq n\left(a_{0}(\lambda-a)\right)=\tau(q(\lambda))=\tau\left(q\left(1-\lambda a_{0}\right)^{-1}\right)=\tau(q)=n(a) .
$$

In precisely the same manner it follows that $d(\lambda-a) \leq d(a)$.

To prove that the indices $n$ and $d$ are constant in a reduced neighbourhood of 0 , it is only necessary to prove it for one of them. Let $x \in N_{r}(\lambda-a)$; then $a^{n} x=\lambda^{n} x$ for all $n \in \mathbb{N}$. Hence, for $\lambda \neq 0$, we have $N_{r}(\lambda-a) \subset$ $\bigcap_{n=1}^{\infty} a^{n} A$. Since $a \in \Phi(A, I)$ we deduce from $a A=(1-p) A$ that $a A$ is a closed right ideal in $A$. But $a^{n} \in \Phi(A, I)$ and so $a^{n} A$ is also closed for all $n \in \mathbb{N}$. Hence, $\widetilde{A}:=\bigcap_{n=1}^{\infty} a^{n} A$ is a closed right ideal in $A$. From $a A \supset a^{2} A \supset \cdots \supset \widetilde{A}$ it follows that if $z \in \widetilde{A}$ then $a z \in a^{n} A$ for all $n$ and we have $a \widetilde{A} \subset \widetilde{A}$. Conversely, $\widetilde{A} \subset a^{n+1} A=a\left(a^{n} A\right)$ for all $n$, from which it follows that

$$
\widetilde{A} \subset \bigcap_{n=1}^{\infty} a\left(a^{n} A\right)=a \bigcap_{n=1}^{\infty} a^{n} A=a \widetilde{A} .
$$

Therefore, $a \widetilde{A}=\widetilde{A}$.

Let $T_{a} z:=a z$ for all $z \in \widetilde{A}$. Then $T_{a}: \widetilde{A} \rightarrow \widetilde{A}$ is surjective. Also, since $N_{r}(\lambda-a) \subset \widetilde{A}$ we have $N\left(\lambda-T_{a}\right)=N_{r}(\lambda-a)$. Hence, $T_{a}$ is a Fredholm operator on the Banach space $\widetilde{A}$ for which $n\left(\lambda-T_{a}\right)=n(\lambda-a)$. Since $T_{a}$ is surjective, $d\left(T_{a}\right)=0$ and from $d\left(\lambda-T_{a}\right) \leq d\left(T_{a}\right)$ it follows that $d\left(\lambda-T_{a}\right)=0$. Therefore,

$$
n(\lambda-a)=n\left(\lambda-T_{a}\right)=\iota\left(\lambda-T_{a}\right)+d\left(\lambda-T_{a}\right)=\iota\left(\lambda-T_{a}\right) .
$$

But $\iota\left(\lambda-T_{a}\right)$ is constant for all $\lambda$ in a sufficiently small neighbourhood $U$ of 0 , and so $n(\lambda-a)$ is constant on $U \backslash\{0\}$. This completes the proof.

In the notation of the proof of the theorem, we have the following characterization of $N_{r}(\lambda-a)$ :

Corollary 3.22. $N_{r}(\lambda-a)=q(\lambda) A \cap \widetilde{A}$. 
Proof. As remarked in the above proof, $N_{r}(\lambda-a) \subset N_{r}\left(a_{0}(\lambda-a)\right)=$ $q(\lambda) A$, and $N_{r}(\lambda-a) \subset \widetilde{A}$, so $N_{r}(\lambda-a)$ is contained in the intersection. Conversely, for $z \in q(\lambda) A \cap \widetilde{A}$ we have $z \in N_{r}\left(a_{0}(\lambda-a)\right)$ and hence $a_{0}(\lambda-a) z=0$. But $(\lambda-a) \widetilde{A} \subset \widetilde{A}$ and so $(\lambda-a) z \in \widetilde{A} \subset a A=(1-p) A$. It follows that $(1-p)(\lambda-a) z=(\lambda-a) z$. However, $a_{0}(\lambda-a) z=0$ implies $0=a a_{0}(\lambda-a) z=(1-p)(\lambda-a) z=(\lambda-a) z$, so $z \in N_{r}(\lambda-a)$.

We remarked earlier that if $I$ is an inessential ideal in the semisimple Banach algebra $A$, then $I \subset \operatorname{kh}(\operatorname{Soc} A)$. In our present setting we assume that $I$ is a trace ideal contained in $\mathrm{kh}(\operatorname{Soc} A)$. Theorem 3.21 enables us to give an independent proof that $I$ is inessential, without using the proof that $\mathrm{kh}(\operatorname{Soc} A)$ is inessential.

Theorem 3.23. Let $A$ be a unital Banach algebra and let the trace ideal $I$ satisfy $\operatorname{Soc} A \subset I \subset \mathrm{kh}(\operatorname{Soc} A)$. Then $I$ is an inessential ideal.

Proof. It will suffice if we can show for all $a \in I$ that every $0 \neq \lambda_{0} \in$ $\partial \sigma(a, A)$ is an isolated point in $\sigma(a, A)$. The element $\lambda_{0}-a$ is a Fredholm element and so, by the above theorem, there exists an $\epsilon$-neighbourhood of $\lambda_{0}$ such that for all $\mu \neq \lambda_{0}$ in this neighbourhood, $\iota(\mu-a), n(\mu-a)$ and $d(\mu-a)$ are constant. But, since this neighbourhood contains elements of the resolvent set $\varrho(a)$, all these numbers are zero. This implies that for all $\mu \neq \lambda_{0}$ in this neighbourhood, $\mu-a$ is invertible. Thus $\lambda_{0}$ is isolated.

4. The exponential spectrum. A useful property of the spectrum for elements $a, b \in A$, with $A$ a Banach algebra, is that

$$
\sigma(a b) \backslash\{0\}=\sigma(b a) \backslash\{0\} .
$$

Whether this is true in general for the exponential spectrum is still an open question. Some sufficient conditions were proved by Murphy, who investigated the exponential spectrum by means of the index group and an abstract index function. He observed that:

Proposition 4.1 ([19, Proposition 4.3 and Corollary 4.4]). Let $A$ be a Banach algebra and $a, b \in A$. Then each of the following conditions implies that $\varepsilon(a b) \backslash\{0\}=\varepsilon(b a) \backslash\{0\}$ :

(1) Either $a$ or $b$ is a limit of invertible elements of $A$.

(2) A is of topological stable rank one.

Observe that if an element $a$ is a Riesz element relative to a closed inessential ideal then $\varepsilon(a b) \backslash\{0\}=\varepsilon(b a) \backslash\{0\}$. Indeed, if $a$ is Riesz relative to a closed inessential ideal, then by [1, Corollary 5.7.5], $\sigma(a)$ is either finite or a sequence converging to 0 and so $a$ is a limit of invertible elements in $A$. 
We add to these results by considering the commutator of two elements. For $a, b \in A$ we note that

$$
1-b a=1-a b+[a, b] .
$$

If $1-a b \in \operatorname{Exp} A$ and $[a, b] \in \operatorname{Rad} A$, then by the remarks preceding Lemma 2.2 in [15], $1-b a \in \operatorname{Exp} A$. If one views the commutator as an inner derivation, then one finds in [7], [8], [9], [16] and [21] conditions ensuring that the commutator belongs to the radical of the Banach algebra.

Note also that, since $\operatorname{Exp} A$ is open in $A^{-1}$, it is also open in $A$. Hence if $1-a b \in \operatorname{Exp} A$, then there exists an $\epsilon>0$ such if $\|[a, b]\|<\epsilon$ then in view of (4.1), $1-b a \in \operatorname{Exp} A$.

We recall that the centre $Z(A)$ of a Banach algebra $A$ is the set

$$
Z(A):=\{a \in A \mid x a=a x \text { for all } x \in A\} .
$$

Proposition 4.2. Let $A$ be a Banach algebra and $a, b \in A$. If $[a, b] \in$ $Z(A)$ then $1-a b \in \operatorname{Exp} A$ if and only if $1-b a \in \operatorname{Exp} A$.

Proof. If $[a, b] \in Z(A)$, then in particular $a[a, b]=[a, b] a$. By the KleineckeShirokov theorem [1, Theorem 5.1.3], $[a, b] \in \mathrm{QN}(A)$. This together with our assumption implies $[a, b] \in \operatorname{Rad} A$. The conclusion now follows from the remarks above.

Our final result is an application of the index theory developed in Section 3. In this regard we also refer to the remarks preceding [19, Proposition 4.5] which gives the classical result that if $H$ is a Hilbert space and $A$ is the Calkin algebra on $H$ then $\varepsilon(a b) \backslash\{0\}=\varepsilon(b a) \backslash\{0\}$ for $a, b \in A$.

In order to prove our main result in this section, we need some preparation. If $I$ is a closed trace ideal such that $\operatorname{Soc} A \subset I \subset \operatorname{kh}(\operatorname{Soc} A)$ then one can decompose the Fredholm elements relative to $I$ into equivalence classes in the following way:

$$
\Phi(A, I)=\bigcup_{n=-\infty}^{\infty} \iota^{-1}(n) .
$$

In view of the index function being continuous it follows that if $a \in A$ is a limit of invertible elements then $\iota(a)=0$.

Proposition 4.3. Let $A$ be a unital Banach algebra and let the trace ideal $I$ satisfy $\operatorname{Soc} A \subset I \subset \operatorname{kh}(\operatorname{Soc} A)$. If $x \in \Phi(A, I)$ satisfies $x+I \in$ $\operatorname{Exp} A / I$ then $\iota(x)=0$.

Proof. If $x+I \in \operatorname{Exp} A / I$ then by Harte's theorem [1, Theorem 3.3.8] there exists $y \in \operatorname{Exp} A$ such that $y+I=x+I$. Hence $y-x \in I$ and so in view of Proposition 3.7(i), for all $\lambda \in \mathbb{C}$,

$$
\iota(x)=\iota(x+\lambda(y-x)) .
$$

In particular, if $\lambda=1$ then $\iota(x)=\iota(y)=0$ because $\operatorname{Exp} A \subset A^{-1}$. 
If $I$ is a closed ideal in a Banach algebra $A$ denote by $J: A \rightarrow A / I$ the canonical homomorphism $J x=x+I, x \in A$.

THEOREM 4.4. Let $A$ be a semisimple Banach algebra and let $I$ be a closed trace ideal such that $\operatorname{Soc} A \subset I \subset \mathrm{kh}(\operatorname{Soc} A)$. If $J^{-1} \operatorname{Exp} A / I=\iota^{-1}(0)$ then, for all $a, b \in A$,

$$
\varepsilon(a b+I, A / I) \backslash\{0\}=\varepsilon(b a+I, A / I) \backslash\{0\} .
$$

Proof. Note that if $1-a b+I \in \operatorname{Exp} A / I$ then $1-b a+I \in(A / I)^{-1}$, i.e., $1-b a \in \Phi(A, I)$. In view of Proposition 4.3 and Theorem 3.20,

$$
0=\iota(1-a b)=\iota(1-b a) .
$$

This together with our assumption $J^{-1} \operatorname{Exp} A / I=\iota^{-1}(0)$ implies that $1-$ $b a+I \in \operatorname{Exp} A / I$.

The condition $J^{-1} \operatorname{Exp} A / I=\iota^{-1}(0)$ together with the index function being continuous implies that $\iota^{-1}(0)$ is a component of $\Phi(A, I)$. For instance, this condition is satisfied in the algebra $B(H)$ of bounded linear operators on a Hilbert space $H$ and $A$ the Calkin algebra on $H$ (see [12, Theorem $5.35])$.

Acknowledgements. The authors express their thanks to Professor J. Zemánek who drew their attention to the work done in [14].

\section{References}

[1] B. Aupetit, A Primer on Spectral Theory, Universitext, Springer, New York, 1991.

[2] B. Aupetit and H. du T. Mouton, Trace and determinant in Banach algebras, Studia Math. 121 (1996), 115-136.

[3] M. M. Bapela, Riesz theory and Fredholm determinants in Banach algebras, $\mathrm{PhD}$ thesis, Univ. of Pretoria, 1999.

[4] B. A. Barnes, Common operator properties of the linear operators $R S$ and $S R$, Proc. Amer. Math. Soc. 126 (1998), 1055-1061.

[5] B. A. Barnes, G. J. Murphy, M. R. F. Smyth and T. T. West, Riesz and Fredholm Theory in Banach Algebras, Pitman, Boston, 1982.

[6] F. F. Bonsall and J. Duncan, Complete Normed Algebras, Springer, Berlin, 1973.

[7] M. Brešar, Derivations decreasing the spectral radius, Arch. Math. (Basel) 61 (1993), 160-162.

[8] - Derivations of noncommutative Banach algebras II, ibid. 63 (1994), 56-59.

[9] M. Brešar and J. Vukman, Derivations of noncommutative Banach algebras, ibid. 59 (1992), 363-370.

[10] C. R. Caradus, W. E. Pfaffenberger and B. Yood, Calkin Algebras and Algebras of Operators on Banach Spaces, Dekker, New York, 1971.

[11] J. B. Conway, Functions of One Complex Variable, Grad. Texts in Math. 11, Springer, New York, 1978.

[12] R. G. Douglas, Banach Algebra Techniques in Operator Theory, Academic Press, New York, 1972. 
[13] J. J. Grobler, H. Raubenheimer and P. van Eldik, Fredholm theory for operators in an operator ideal with a trace I, Integral Equations Operator Theory 5 (1982), 774-790.

[14] H. Kraljević, S. Suljagić and K. Veselić, Index in semisimple Banach algebras, Glas. Mat. Ser. III 17 (37) (1982), 73-95.

[15] L. Lindeboom (Groenewald) and H. Raubenheimer, Different exponential spectra in Banach algebras, Rocky Mountain J. Math. 29 (1999), 957-970.

[16] M. Mathieu and G. J. Murphy, Derivations mapping into the radical, Arch. Math. (Basel) 57 (1991), 469-474.

[17] H. du T. Mouton, On inessential ideals in Banach algebras, Quaest. Math. 17 (1994), $59-66$.

[18] S. Mouton and H. Raubenheimer, More spectral theory in ordered Banach algebras, Positivity 1 (1997), 305-317.

[19] D. J. Murphy, The index group, the exponential spectrum and some spectral containment theorems, Proc. Roy. Irish Acad. Sect. A 92 (1992), 229-238.

[20] A. Pietsch, Eigenvalues and s-numbers, Cambridge Stud. Adv. Math. 13, Cambridge Univ. Press, Cambridge, 1987.

[21] V. Pták, Commutators in Banach algebras, Proc. Edinburgh Math. Soc. 22 (1979), 207-211.

[22] J. Puhl, The trace of finite and nuclear elements in Banach algebras, Czechoslovak Math. J. 28 (1978), 656-676.

[23] M. Schechter, Principles of Functional Analysis, Academic Press, New York, 1971.

Unit for Business Mathematics and Informatics

North-West University

Potchefstroom 2520, South Africa

E-mail: Koos.Grobler@nwu.ac.za
Department of Mathematics University of Johannesburg Johannesburg 2006, South Africa E-mail: heinrichr@uj.ac.za

Received December 14, 2007

Revised version April 7, 2008 